\title{
IL LASER: FULCRO DI GRANDI INFRASTRUTTURE DI RICERCA
}

\author{
Nota del s.c. SANDRO DE SILVESTRI (*)
}

(Adunanza del 27 novembre 2014)

SUNTO. - Una caratterista importante delle sorgenti laser consiste nella capacità di generare brevi e intensi impulsi di radiazione. Tale caratteristica ha stimolato la realizzazione di infrastrutture laser su larga scala principalmente dedicate alla fusione nucleare. Il presente lavoro presenta la realizzazione di una nuova infrastruttura di ricerca "Extreme Light Infrastructure" (ELI), concettualmente differente e con finalità diverse dalle precedenti. La caratteristica principale consiste essenzialmente nella produzione di potenze di picco estremamente elevate utilizzando impulsi della durata di pochi femtosecondi ed di alta energia. Focalizzando tali impulsi su di un'area delle dimensioni della lunghezza d'onda si potranno così ottenere intensità estremamente elevate, che consentiranno nuove ricerche nella fisica delle particelle, nella fisica gravitazionale, nella teoria non lineare dei campi, nella fisica delle altissime pressioni, nell'astrofisica e cosmologia.

$* * *$

ABSTRACT. - An important characteristic of the laser sources consists in the ability to generate short and intense radiation pulses. This feature has stimulated the development of large-scale laser infrastructures mainly dedicated to nuclear fusion. This paper presents the realization of a new research infrastructure "Extreme Light Infrastructure" (ELI) which has conceptually different purposes compared to the others. The main feature consists essentially in the production of an extremely high peak power by using pulses of the duration of a few femtoseconds and of high energy. Focusing these pulses over an area of the size of the laser wavelength one can achieve extremely high intensities, which will enable new research in particle physics, gravitational physics, nonlinear field theory, physics of high pressures, astrophysics and cosmology.

Milano, Italy.

Dipartimento di Fisica, Politecnico di Milano, Piazza L. da Vinci 32, 20133

E-mail: sandro-desilvestri@polimi.it 


\section{INTRODUZIONE}

L'invenzione del laser con la prima dimostrazione avvenuta nel 1960 ha inciso profondamente nello sviluppo tecnologico e scientifico grazie alla capacità di controllare la coerenza (sia spaziale che temporale) della radiazione emessa. Questa è la differenza sostanziale tra la luce emessa da una tradizionale sorgente di luce e quella emessa da un laser. Numerose sono le applicazioni della radiazione laser che parallelamente allo sviluppo delle sorgenti si sono via via affermate con successo [1] . Le lavorazioni meccaniche come il taglio, la foratura e la saldatura vengono realizzate con laser a gas, a stato solido e in fibra con efficienze e qualità superiori a quelle ottenibili mediante tecniche tradizionali. Nel campo delle comunicazioni ottiche vengono utilizzati i laser a semiconduttore, operanti alle lunghezze d'onda dell'infrarosso corrispondenti alla condizione di bassa attenuazione e minima dispersione delle fibre ottiche, che sono sorgenti ideali grazie alla loro compattezza e basso consumo energetico. Per quest'ultime caratteristiche i laser a semiconduttore sono anche utilizzati nei lettori di CD e DVD. In medicina branche come la chirurgia, la fototerapia, la fotoablazione e la diagnostica trovano nell'uso delle sorgenti laser la possibilità di nuove terapie e metodiche di indagine poco invasive. Nel settore della metrologia, l'utilizzo dei laser consente il riconoscimento selettivo e la misura della concentrazione di composti chimici con applicazioni interessanti nel monitoraggio ambientale per il controllo anche degli agenti inquinanti.

Una ulteriore importante caratteristica delle sorgenti laser consiste nella capacità di generare brevi e intensi impulsi di radiazione. Tale caratteristica ha stimolato la realizzazione di infrastrutture laser su larga scala la cui motivazione principale consisteva nelle ricerche sulla fusione nucleare via laser per la produzione di energia. In questa categoria le infrastrutture più avanzate sono rappresentate attualmente dalla "National Ignition Facility" (NIF) negli Stati Uniti e dal "Laser Megajoule" in Francia. NIF ad esempio fornisce un'energia di circa un $\mathrm{MJ}$ in pochi nanosecondi $\left(1 \mathrm{~ns}=10^{-9} \mathrm{~s}\right)$ a cui corrisponde una potenza di picco di 0.5 PW. Nel 2007 è stata proposta la "Extreme Light Infrastructure" (ELI) nell'ambito della roadmap dell'European Science Forum for Research Infrastructures (ESFRI), un organismo dell'Unione Europea per il coordinamento delle infrastrutture di ricerca in Europa. Si tratta di un nuovo tipo di infrastruttura laser, concet- 
tualmente differente e con finalità diverse dalle precedenti, la cui caratteristica principale consiste essenzialmente nella produzione di potenze di picco estremamente elevate con l'intenzione di raggiungere il regime sub-Exawatt, corrispondente a circa 1000 volte la potenza sviluppata da NIF e da "Laser Megajoule". Inoltre ELI sarà totalmente aperta alla comunità internazionale degli utilizzatori.

\section{ELI: IMPULSI LASER ULTRABREVI E INTENSI}

L'idea di base per ottenere i livelli di potenza previsti in ELI, consiste nel concentrare l'energia della radiazione laser in impulsi estremamente brevi nel tempo. Questo è reso possibile grazie al continuo sviluppo di sorgenti laser in grado di produrre impulsi con durate via via più brevi dai picosecondi $\left(1 \mathrm{ps}=10^{-12} \mathrm{~s}\right)$ ai femtosecondi $\left(1 \mathrm{fs}=10^{-15} \mathrm{~s}\right)$. Il campo elettrico della luce visibile oscilla con un periodo di 2 femtosecondi, ossia 500000 miliardi di volte in un secondo. E' possibile generare impulsi di luce laser ultrabrevi in grado di contenere solo pochi cicli di oscillazione [2]. Parallelamente, lo sviluppo della tecnica di amplificazione degli impulsi, denominata "Chirped Pulse Amplification" [3], ha consentito nel corso degli anni di poter aumentarne notevolmente l'energia. Focalizzando impulsi ultrabrevi e amplificati su di un'area delle dimensioni della lunghezza d'onda laser (pochi micrometri di diametro) sono state ottenute intensità estremamente elevate dell'ordine di $10^{22}$ $\mathrm{W} / \mathrm{cm}^{2}$ [4]. Le intensità che si vogliono raggiungere con ELI sono almeno 3-4 ordini di grandezza superiori a quelle disponibili attualmente. Tali intensità sono anche dette ultrarelativistiche poiché i relativi campi sono in grado di accelerare non solo gli elettroni ma anche gli ioni a velocità prossime a quelle della luce.

ELI vuole rappresentare un approccio completamente nuovo allo studio della fisica fondamentale basato su caratteristiche uniche, quali ad esempio:

- Il più elevato campo elettrico.

- La possibilità di controllare il moto a velocità relativistiche dei costituenti fondamentali della materia (ioni ed elettroni).

- La generazione di radiazione coerente e incoerente di alta energia come raggi $\mathrm{X}$ o raggi gamma.

- La possibilità di produrre gli impulsi di luce più brevi mai realizzati consentendo di superare il dominio temporale degli attosecon- 
di $\left(1 \mathrm{as}=10^{-18} \mathrm{~s}\right)$ fino ad arrivare, in prospettiva, a quello degli zeptosecondi $\left(1 \mathrm{zs}=10^{-21} \mathrm{~s}\right)$.

Queste caratteristiche da sole o combinate consentono di realizzare un nuovo insieme di potenti strumenti di indagine per diverse branche della scienza. La visione a lungo termine di ELI consiste nel portare l'uso del laser in quei settori in cui era assente dando impulso a nuove aree della fisica, come ad esempio: (i) l'elettrodinamica quantistica non lineare in presenza di campi laser intensi; (ii) l'accelerazione di particelle basata sulla radiazione laser; (iii) nuovi modi di produrre radiazione a lunghezze d'onda ultracorte nella regione dei raggi $\mathrm{X}$ duri e gamma associata alla disponibilità di tale radiazione sotto forma di impulsi ultrabrevi. Una rassegna completa di tutta l'attività prevista per ELI sia per quanto riguarda lo sviluppo delle sorgenti e il relativo caso scientifico è riportata nel riferimento bibliografico [5].

\section{ELI: APPLICAZIONI}

ELI consentirà nuove ricerche nella fisica delle particelle, nella fisica gravitazionale, nella teoria non lineare dei campi, nella fisica delle altissime pressioni, nell'astrofisica e cosmologia. Accanto alla sua missione in fisica fondamentale un importante obiettivo di ELI consiste nella generazione di fasci ultrabrevi di particelle energetiche (10-100 $\mathrm{GeV}$ ) e radiazione (sino a qualche $\mathrm{MeV}$ ) prodotti da compatti acceleratori laser-plasma. ELI imposterà la sua missione scientifica, tecnica e medica a beneficio dell'industria e più in generale della società. Accanto alla realizzazione della sorgente laser principale sub-exawatt, lo sviluppo di sorgenti di luce secondarie consentirà di disporre di tecnologie a raggi $\mathrm{X}$ per chiarire l'evoluzione temporale delle reazioni quali l'attività e il folding delle proteine, la radiolisi, il monitoraggio dei legami chimici e i processi di catalisi. Questo porterà ad una migliore comprensione e controllo di eventi chiave durante la formazione e la rottura dei legami chimici. Ci si aspetta un forte impatto sulla società e sulle nuove tecnologie per l'industria poiché questi processi giocheranno un ruolo importante nella produzione di nuovi farmaci e nel miglioramento della loro efficienza. La disponibilità di una sorgente gamma intensa e accordabile consentirà ad esempio la produzione di nuovi radioisotopi per la medicina per determinare l'efficienza della chemioterapia dei tumori e la dose ottimale per l'imaging nucleare. Nuove cop- 
pie di radioisotopi per lo stesso elemento potranno rendersi disponibili, uno per la diagnostica e l'altro per la terapia, consentendo di controllare e ottimizzare il trasporto dell'isotopo attraverso il proprio elemento bio-coniugato nel tumore. Emettitori di elettroni Auger di bassa energia per una efficiente e mirata terapia tumorale potranno essere prodotti per uso clinico. La ricerca medica in questa direzione aprirà nuove prospettive per la società. Nel campo della scienza dei materiali ELI aiuterà a chiarire i meccanismi che portano alla creazione dei difetti e al deperimento dei materiali nei reattori nucleari. Occorre inoltre sottolineare che i fasci di radiazione e di particelle prodotte dai laser di ELI risulteranno perfettamente sincronizzati tra di loro per il modo con cui vengono generati dagli impulsi ottici di alta intensità. Questo consente l'uso di tecniche miste di pump-probe in un intervallo estremamente ampio di energie dei fotoni $(\mathrm{eV}-\mathrm{MeV})$ e fasci di particelle $(\mathrm{eV}-\mathrm{GeV})$ per sperimentazioni finora inedite.

In una visione a lungo termine ELI potrà diventare la più avanzata infrastruttura laser in campo internazionale per gli utilizzatori, promuovendo la scienza fondamentale e le sue applicazioni in molte aree di rilevanza sociale attraverso la disponibilità di sorgenti laser ultraintense.

\section{ELI: IMPLEMENTAZIONE}

La realizzazione di ELI passa attraverso tre fasi distinte. La prima fase è iniziata nel 2007 con un finanziamento da parte dell'Unione Europea di $6 \mathrm{M} €$ dedicato alla "Preparatory Phase" di ELI (ELI-PP). Si trattava di un progetto della durata di 3 anni, sostenuto da oltre 40 istituzioni di ricerca provenienti da 13 paesi europei, con il compito di portare ELI a un livello di maturità scientifica, organizzativa e finanziaria. La partecipazione italiana alla "Preparatory Phase" di ELI è stata coordinata dal CNR e dall'INFN. Durante i lavori il caso scientifico di ELI si è espanso notevolmente prendendo atto che le enormi potenzialità di ELI non potevano essere coperte da una singola infrastruttura. Nell'ottobre 2009 lo Steering Committee della "Preparatory Phase" ha dato il mandato alla Romania, Repubblica Ceca e Ungheria di implementare congiuntamente il progetto ELI attraverso la costruzione di un'infrastruttura distribuita con tre sedi coordinate e con missioni diverse: (i) applicazioni di fisica nucleare (RO); (ii) fasci di particelle 
secondarie (elettroni e ioni) (CZ); (iii) impulsi ad attosecondi (HU), che rappresentano le "Grand Challenges" identificati nel caso scientifico del progetto [5]. Qui di seguito vengono sintetizzati gli obiettivi tecnologici e scientifici delle tre sedi:

- ELI-Nuclear Physics(ELI-NP) (Magurele, Romania): dedicata allo sviluppo di laser ultraintensi e alla generazione di fasci gamma a banda stretta e accordabili per ricerche di frontiera nell'ambito della fisica nucleare (http://www.eli-np.ro/).

- ELI-Beamlines (Praga, Repubblica Ceca): dedicata alla generazione di pacchetti impulsivi di elettroni fino a $10 \mathrm{GeV}$, protoni fino a qualche centinaio di $\mathrm{MeV}$ e radiazione $\mathrm{X}$ fino a qualche $\mathrm{MeV}$ prodotta da acceleratori compatti a laser-plasma (http://www.eli-beams.eu/).

- ELI-Attosecond Light Pulse Source (ELI-ALPS) (Szeged, Ungheria): dedicata allo sviluppo di sorgenti laser ultrabrevi nella regione spettrale dei raggi $\mathrm{X}$ con durate sino al regime degli attosecondi per lo studio di dinamiche elettroniche in atomi, molecole, plasmi e solidi (http://www.eli-hu.hu/).

Le tre sedi saranno dotate di sorgenti laser multi-petawatt con caratteristiche diverse. Il costo complessivo stimato per la costruzione di ELI è pari a circa $800 \mathrm{M} €$, cosi distribuito: (i) la Repubblica Ceca (ELI-Beamlines) investirà circa $270 \mathrm{M} €$; (ii) la Romania (ELI-NP) circa $280 \mathrm{M} €$; (iii) l'Ungheria (ELI-ALPS) circa $260 \mathrm{M} €$. Il modello di finanziamento adottato per la realizzazione di ELI è del tutto pioneristico in quanto prevede per la sua quasi totalità l'utilizzo dei fondi strutturali che l'Unione Europea ha assegnato alle nazioni coinvolte. In generale i fondi strutturali vengono erogati per potenziare e sviluppare attività in regioni che necessitano la costruzione di infrastrutture. ELI rappresenta il primo caso in cui i fondi strutturali vengono utilizzati per finanziare infrastrutture di ricerca. In realtà la presenza di una infrastruttura ad alto contenuto tecnologico aperta alla comunità internazionale determina una importante ricaduta anche a livello regionale essendo di stimolo alle industrie e università sul territorio. La realizzazione dell'infrastruttura è prevista per la fine del 2018.

Al termine della "Preparatory Phase", a partire dal 2011, ha fatto seguito la costituzione di un'entità legale (ELI-DC, Delivery Consortium, http://www.eli-laser.eu/) che vede inizialmente presenti oltre ai tre stati coinvolti nella costruzione di ELI l'Italia, la Gran Bretagna e la Germania. Lo scopo di ELI-DC consiste nel: (i) coordinare l'implementazione dei tre siti garantendo la complementarietà 
della loro missione; (ii) supportare l'implementazione di ELI attraverso un uso coordinato delle risorse umane e dei mezzi tecnologici e finanziari disponibili a livello europeo; (iii) definire le condizioni per l'implicazione e la contribuzione degli altri paesi dell'UE; (iv) organizzare le negoziazioni per diventare a partire dal 2018 un "European Research Infrastructure Consortium" (ELI-ERIC), entità legale creata dalla UE per facilitare la formazione di consorzi che realizzino e gestiscano infrastrutture di ricerca paneuropee di grandi dimensioni. La partecipazione delle nazioni europee ad ELI-DC si sta costituendo mediante adesioni ed espressioni di interesse a diversi livelli.

\section{ELI: PARTECIPAZIONE ITALIANA}

L'Italia partecipa ad ELI-DC ed ha interessi scientifici forti sia nella realizzazione sia nell'utilizzo della strumentazione presenti nelle sedi dell'infrastruttura. Gli enti di ricerca quali INFN, CNR e ElettraST hanno notevoli competenze in diversi settori tecnologici che riguardano le infrastrutture previste per ELI. In particolare dispongono di competenze progettuali e capacità costruttive nel settore degli acceleratori, dei sistemi laser a impulsi ultrabrevi di elevata energia, nonché nella realizzazione e nella gestione di grandi infrastrutture di ricerca aperte all'utenza internazionale. I tre Enti hanno forti connessioni tra di loro e con gruppi di ricerca universitari e contano su una qualificata capacità produttiva industriale. In particolare, l'INFN sta coordinando il consorzio EuroGammaS, che coinvolge 12 partner tra istituti e aziende europei, per la realizzazione della sorgente gamma a elevata intensità prevista per ELI-NP e collabora attivamente con ELI-Beamlines (sulla produzione di sorgenti secondarie di protoni per uso medicale). Il CNR ha attivato una collaborazione con ELI-ALPS per la progettazione e realizzazione di due beamline per l'utilizzo di impulsi ad attosecondi per applicazioni in fisica atomica e molecolare e ha anche connessioni con ELI-Beamlines per lo sviluppo di sistemi di accelerazione di particelle e strumentazione per laser di potenza. Elettra-ST ha interessi rilevanti in ELI, avendo sviluppato un vasto know-how nei sistemi per stabilizzazione, sincronizzazione e trasporto d'impulsi ultrabrevi, nell'ambito delle ottiche X-UV, nei sistemi a radiofrequenza in banda $\mathrm{X}$ e in generale nella diagnostica degli acceleratori. Infine per l'utilizzo futuro delle potenzialità offerte dalle sorgenti previste in ELI, vi è una forte 
comunità scientifica nazionale con competenze multidisciplinari e specifiche per ciascuna delle tre sedi.

\section{CONCLUSIONI}

ELI rappresenta una irripetibile occasione per la comunità scientifica internazionale e in particolare per l'Europa di poter disporre di una infrastruttura tecnologicamente avanzata nel campo delle sorgenti laser e delle relative applicazioni in diversi campi della scienza. In particolare a livello nazionale si assiste ad una importante opportunità da cogliere in quanto parte della programmazione della ricerca in atto nei tre enti nazionali convolti è indirizzata da tempo su tematiche fortemente connesse a quelle previste per ELI, ovviamente su un diverso fattore di scala. Essendo ormai entrati nella fase di implementazione di ELI e in vista dell'ampio spettro di attività di ricerca nelle tre sedi, il contributo di INFN, CNR e Elettra-ST diventa un naturale sviluppo dell'attuale contesto e assume un'importanza strategica in particolare per l'elevato valore tecnologico presente nella strumentazione da realizzare. Si renderà inoltre necessario produrre uno sforzo organico da parte dei tre enti e delle università italiane nella formazione di personale qualificato per la gestione della strumentazione operante in ELI e nella formazione della comunità degli utilizzatori.

\section{BIBLIOGRAFIA}

[1] O. Svelto, "Principles of lasers", 5th ed., Springer (2010).

[2] M. Nisoli, S. De Silvestri, O. Svelto, R. Szipöcs, K. Ferencz, Ch. Spielmann, S. Sartania, and F. Krausz, "Compression of high-energy laser pulses below 5 fs", Opt. Lett. 22, 522 (1997).

[3] D. Strickland and G. Mourou, "Compression of amplified chirped optical pulses", Opt. Commun. 56, 219 (1985).

[4] V. Yanovsky, V. Chvykov, G. Kalinchenko, P. Rousseau, T. Planchon, T. Matsuoka, A. Maksimchuk, J. Nees, G. Cheriaux, G. Mourou, and K. Krushelnick, "Ultrahigh intensity-300-TW laser at $0.1 \mathrm{~Hz}$ repetition rate", Opt. Express 16, 2109 (2008).

[5] ELI White Book, "Science and Technology with High Intensity Lasers", ed. by G. Mourou and G. Korn, http://www.eli-beams.eu/wp-content/uploads/2011/08/ ELI-Book_neues_Logo-edited-web.pdf 